\title{
LncRNA RNCR2 facilitates cell proliferation and epithelial-mesenchymal transition in melanoma through the HK2-mediated Warburg effect via targeting miR-495-3p
}

\author{
Bao-Xue DUAN, Xue-Rui GENG ${ }^{*}$, Yao-Qun WU* \\ Department of Dermatology, Xiangyang No.1 People’s Hospital, Hubei University of Medicine, Xiangyang 441000, Hubei Province, P.R.C \\ *Correspondence: yaaquu789@163.com \\ \#Contributed equally to this work.
}

Received November 20, 2020 / Accepted February 2, 2021

\begin{abstract}
Melanoma is a potentially lethal skin cancer with a high death rate. LncRNAs were reported to be implicated in melanoma progression. However, the function and mechanisms of lncRNA RNCR2 in melanoma are little known. In this study, RNCR2, miR-495-3p, and HK2 expression levels were measured in melanoma tissue specimens and cell lines by qPCR. EdU and CCK-8 assays were performed to assess cell proliferation. Enolase activity, ATP level, lactate production, and glucose consumption measurement kits were used to evaluate the glycolysis of tumor cells. Immunofluorescence and western blot were used to detect the expression of epithelial-mesenchymal transition (EMT) and glycolysis-related proteins. Luciferase reporter assay was applied to confirm the target relationships. The role of RNCR2 in tumorigenesis was examined using murine xenograft models. LncRNA RNCR2 was upregulated in melanoma tissues and cell lines. Cell function detection showed that RNCR2 knockdown remarkably inhibited cell proliferation and EMT via glycolysis, as well as reduced the growth of a tumor. Mechanically, RNCR2 was confirmed to bind to miR-495-3p and positively regulated HK2 expression level, and the miR-495-3p level was negatively correlated with RNCR2 or HK2 in melanoma tissues. Further, miR-495-3p downregulation or HK2 upregulation partially reversed RNCR2 knockdown-induced inhibition of melanoma cell growth, EMT, and glycolysis. Collectively, RNCR2 might be an oncogenic lncRNA to promote tumor cell glycolysis and accelerate tumor growth via the miR-495-3p/HK2 axis, providing a promising treatment target for melanoma.
\end{abstract}

Key words: melanoma, lncRNA RNCR2, miR-495-3p, HK2, Warburg effect

Melanoma is a type of aggressive skin cancer, and the global incidence of melanoma is rapidly increasing every year [1-3]. Although many efforts have been made to treat melanoma (surgery, chemotherapy, targeted drugs, and immunotherapy), the curative effect and survival rate have not been markedly improved [4-7]. Therefore, it is urgent to explore molecular mechanisms of melanoma carcinogenesis, which are essential to finding new therapeutic targets and strategies for melanoma.

In tumors and other cells with abnormal proliferation or development, abnormal glucose metabolism is one of the most obvious features [8]. Compared with surrounding tissues, tumor cells absorb a lot of glucose. Moreover, even in the case of sufficient oxygen, the glycolysis process that produces lactic acid occurs in tumor cells. This aerobic glycolysis is also called the Warburg effect $[9,10]$. Compared with normal cells, due to the increased glucose uptake and glycol- ysis of cancer cells, the production of intermediate metabolites and end products of glycolysis, pyruvate, also increases [11]. This characteristic change in metabolism is to meet the biosynthetic requirements of uncontrolled proliferation of tumor cells [12]. Epithelial-mesenchymal transition (EMT) is the main cause of tumorigenesis, malignant transformation, and metastasis. It is worth noting that the enhancement of aerobic glycolysis in tumor cells can promote stemness and EMT [13, 14]. Therefore, targeted aerobic glycolysis is of great significance for the treatment and prevention of tumors.

Long non-coding RNA (lncRNA) is a highly heterogeneous group of RNAs that does not encode proteins and whose number of nucleotides exceeds 200. It is mainly expressed in the nucleus and highly conserved in mammals $[15,16]$. LncRNA is believed to play a special role in certain malignant tumors by regulating protein molecular signals 
and cell functions [17, 18]. Studies have found that the expression of a variety of lncRNAs in melanoma promotes the occurrence of cancer, and is closely related to aerobic glycolysis and EMT in tumor development [19-24]. Retinal non-coding RNA 2 (RNCR2) is a type of IncRNAs associated with the risk of myocardial infarction and is also expressed in retinal tissues and nerve cells [25-27]. In addition, it has been found that lncRNA RNCR2 has a regulatory relationship with miR-150 and PI3K/AKT signaling pathways in melanoma cells to promote melanoma cell proliferation and invasion $[28,29]$. However, the roles and mechanisms of RNCR2 in regulating glycolysis have not been revealed. In the previous studies, hexokinase 2 (HK2) is a rate-limiting enzyme for glycolysis, which is highly expressed in many human cancers to promote proliferation, angiogenesis, and glycolysis [30, 31]. Nevertheless, whether lncRNA RNCR2 could modulate proliferation and EMT in melanoma by regulating HK2-mediated glycolysis still remains unknown.

In this study, we demonstrated that lncRNA RNCR2 acted as a contributor for melanoma cell proliferation and EMT via modulating glycolysis, which is remarkably reversed by the miR-495-3p/HK2 axis. Therefore, we proposed a novel mechanism by which lncRNA RNCR2 promoted glycolysis, proliferation, and EMT in melanoma cells, which would help make crucial strategies for treating melanoma.

\section{Materials and methods}

Collection of clinical tissue samples. Melanoma tissues and matched adjacent normal tissues were obtained from 35 melanoma patients who received consent for surgery, and all participating patients signed informed consent. The patients treated by chemotherapy or radiation therapy were screened out. The experiments were conducted with the approval of the ethics committee of Xiangyang No.1 People's Hospital, Hubei University of Medicine.

Cell culture. Melanoma cell lines (A375, MUM2B, B16, SK-MEL-1, A2058) and human epidermal melanocyte HEMa-LP were purchased from the ATCC (USA) and cultured in DMEM (Gibco, USA) with 10\% FBS (Invitrogen, USA) at $37^{\circ} \mathrm{C}$ with $5 \% \mathrm{CO}_{2}$.

Plasmids and transfection. miR-495-3p mimics, miR-495-3p inhibitors, RNCR2 shRNA (sh-RNCR2), and the negative controls (miR-NC, inhibitor NC, sh-NC) were chemically synthesized by GenePharma (Shanghai, China). For HK2 overexpression, the cDNA of HK2 was cloned into a pcDNA3.1 vector (Invitrogen). Cells were transfected with plasmids by using Lipofectamine 3000 (Invitrogen).

Quantitative real-time PCR (qPCR). The TRIzol reagent (Invitrogen) was used to extract total RNA according to specified procedures. Then, total RNA was reversely transcribed into complementary DNA (cDNA), followed by qPCR examination using SYBR Green Master Mix on the ABI 7900 RealTime PCR System. Relative lncRNA RNCR, miR-495-3p, and HK2 expression levels were calculated by using $2^{-\Delta \Delta C t}$ method and were normalized to GAPDH or U6. Primers were listed as follows: RNCR2 5'-TCCCATTCCCGGAAGCTAGA-3' (sense), 5'-GAGGCATGAAATCACCCCCA-3' (anti-sense); miR-495-5p 5'-GGCGAAACAAACATGGTGCA-3' (sense), 5'-CGGCCCAGTGTTCAGACTAC-3' (anti-sense); HK2 5'-CCTCGGTTTCCCAACTCTG-3' (sense), 5'-GGTAGAGATACTGGTCAACCTTC-3' (antisense); GAPDH 5'-AGGTCGGAGTCAACGGATTT-3' (sense), 5'-TGACGGTGCCATGGAATTTG-3' (antisense); U6 5'-CTCGCTTCGGCAGCACA-3' (sense), 5'-AACGCTTCACGAATTTGCGT-3' (anti-sense).

Cell proliferation. Transfected melanoma cells $\left(5 \times 10^{3} /\right.$ well) were seeded into 96-well plates overnight, and then $10 \mu$ l CCK- 8 solution (Beyotime, Shanghai, China) incubated with cells was removed at the indicated time for $2 \mathrm{~h}$. Finally, the absorbance of cells ( $450 \mathrm{~nm}$ ) was measured on a microplate reader. For 5-ethynyl-2'-deoxyuridine (EdU) assay, a measurement kit (RiboBio, Guangzhou, China) was used to evaluate the synthesis of DNA in growing melanoma cells according to the instructions. Lastly, a fluorescence microscope was used to observe the immunostaining of EdU or DAPI and count EdU-positive cells.

Glucose consumption and lactate production measurement. After the transfection, melanoma cells were cultured in 6-well plates for $12 \mathrm{~h}$. Glucose measurement and lactate assay kits (Sigma-Aldrich, USA) were used to assess glucose consumption and lactate production according to the manufacturer's instructions.

Enolase activity and ATP level determination. The levels of ATP and enolase activity were assessed by using the Enhanced ATP assay kit (Beyotime) and Enolase Activity Assay Kit (Sigma-Aldrich). The analysis process corresponds to the instructions attached to each kit.

Measurement of Glucose-6-Phosphate (G6P) level. The level of G6P in melanoma cells was detected by using a G6P Assay Kit (Beyotime, Shanghai, China), as directed by the manufacturer's instructions.

Bioinformatics analysis and dual luciferase assay. The interaction of miR-495-3p with RNCR2 or HK2 was predicted using starBase (http://starbase.sysu.edu.cn/). RNCR2 or HK2 fragments with wild-type (WT) or mutant (MUT) miR-495-3p binding sequences were obtained from GenePharma. The WT and MUT sequences were inserted into the psi-CHECK2 luciferase reporter vector (Promega, Madison, Wisconsin, USA) and named RNCR2-WT/HK2-WT and RNCR2-MUT/HK2-MUT, respectively. The miR-495-3p mimics or miR-NC plus RNCR2-WT/HK2-WT or RNCR2MUT/HK2-MUT was transfected into melanoma cells with the help of Lipofectamine 3000. The cells were harvested after $48 \mathrm{~h}$ of transfection, and dual-luciferase reporter gene assay system (Promega) was performed to test the luciferase activity. Luciferase activity was normalized to Renilla luciferase activity.

RNA immunoprecipitation (RIP) analysis. Magna RIP RNA binding protein immunoprecipitation kit (Millipore, 
Burlington, MA, USA) was used to confirm the relation between RNCR2 and miR-495-3p. Firstly, the cells were lysed in the RIP lysis buffer. Anti-AGO2 antibody and control IgG (Millipore) were coupled with magnetic beads and incubated with cell extracts at $4{ }^{\circ} \mathrm{C}$ overnight. Then, magnetic beads were collected, washed, and digested with proteinase $\mathrm{K}$. Finally, the immunoprecipitated RNA was extracted and analyzed by qPCR to evaluate the enrichment of RNCR 2 and miR-495-3p on the magnetic beads containing AGO2.

Western blot analysis. Total proteins were prepared using RIPA lysis buffer. Then, equal amounts $(20 \mu \mathrm{g})$ of protein from each group were separated by SDS-PAGE electrophoresis and transferred to polyvinylidene fluoride (PVDF) membranes. The PVDF membranes were blocked with nonfat milk for $1 \mathrm{~h}$ and then soaked with primary antibodies: E-cadherin (1:20,000; ab40772, Abcam, MA, USA), $\mathrm{N}$-cadherin (1:1,000; ab18203, Abcam), Vimentin (1:2,000; ab92547, Abcam), Snail (1:500; ab82846, Abcam), and HK2 (1:10,000; ab227198, Abcam) at $4^{\circ} \mathrm{C}$ overnight. After washing in TBST, membranes were incubated with horseradish peroxidase (HRP)-conjugated secondary antibody for $2 \mathrm{~h}$. Proteins were visualized using enhanced chemiluminescence (ECL) kit (Jiancheng, Nanjing, China) and quantified by ImageJ software.

Immunofluorescence analysis. Cells from different treatment groups were collected and fixed with $4 \%$ paraformaldehyde for $15 \mathrm{~min}$. Subsequently, cells were permeabilized with $0.05 \%$ Triton X-100 for 10 minutes and blocked with 5\% BSA for $1 \mathrm{~h}$. Then the cells were combined with primary antibody E-cadherin (1:500; ab40772, Abcam) and N-cadherin (1:200; ab18203, Abcam) overnight at $4{ }^{\circ} \mathrm{C}$, and then incubated with the anti-rabbit Alexa Fluor 488 in the dark for 1 h. Lastly, nuclear staining was performed by using DAPI (Solarbio, Beijing, China). The cells were examined and photographed with a fluorescence microscope.

Murine xenograft model. Eight BALB/c nude mice (4-6 weeks old) were obtained from SLAC Laboratory Animals Co., Ltd. (Shanghai, China). For tumor xenograft assay, a total of $1 \times 10^{7}$ A375 cells stably transfected with sh-RNCR2 or sh-NC were subcutaneously injected into the mice. Tumor size was calculated every 4 days by the formula: $\mathrm{V}=\left(\mathrm{L} \times \mathrm{W}^{2}\right) / 2$ (V: volume, L: length, W: width). The mice were sacrificed at the designated time and the tumors were collected, weighted, and saved for further experiments. The animal study was approved and monitored by the ethics committee of Xiangyang No.1 People's Hospital, Hubei University of Medicine.

Statistical analysis. Student's t-test was used for two groups comparison and the differences between multiple groups were compared by one-way analysis of variance (ANOVA). Spearman's correlation analysis was performed to assess the correlation between levels of miR-495-3p and RNCR2 or HK2 in melanoma patient tissues. All data were statistically evaluated using GraphPad Prism 6.0 software. The experiments were conducted at least three replications, and the data were presented as mean $\pm \mathrm{SD}$.

\section{Results}

LncRNA RNCR2 is upregulated in melanoma. To analyze the potential role of RNCR2 in melanoma progression, $\mathrm{qPCR}$ was performed to measure RNCR2 level. The data revealed that RNCR2 was upregulated in melanomas (Figure 1A). Subsequently, RNCR2 levels in A375, MUM2B, B16, SK-MEL-1, and A2058 cells were measured. The results showed that RNCR2 was highly expressed in melanoma cells, especially A375 and A2058, in comparison with normal human epidermal melanocytes (Figure 1B). Taken together, dysregulation of lncRNA RNCR2 might be involved in melanoma development.

Knockdown of RNCR2 inhibits melanoma cell proliferation and EMT via modulating glycolysis. To explore the effect of RNCR2 on the proliferation and EMT of melanoma cells, A375 and A2058 cells were transfected with sh-NC or sh-RNCR2 followed by the validation of transfection efficiency (Figure 2A). Cell proliferation assays exhibited that the inhibition of RNCR2 drastically decreased cell viability (Figure 2B) and EdU-positive cells (Figure 2C). In addition, deficiency of RNCR2 distinctly downregulated N-cadherin, Vimentin, and Snail expression, while promoted E-cadherin level (Figure 2D). Photographs of immunofluorescence
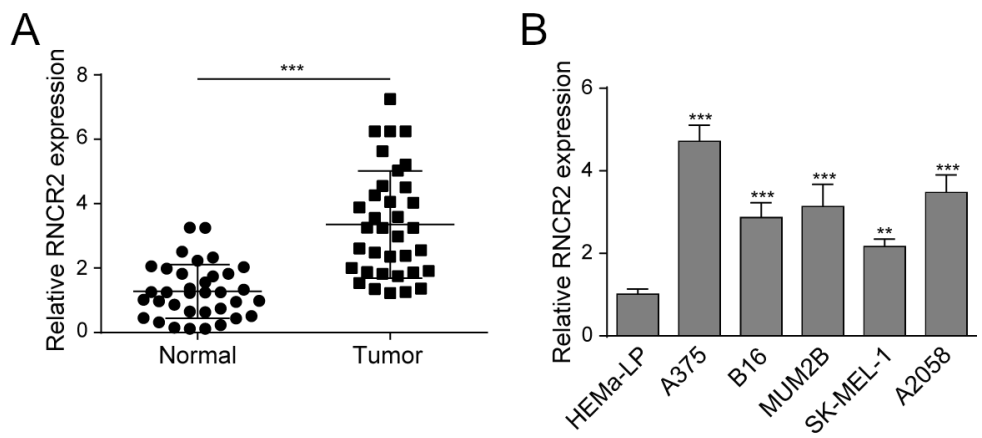

Figure 1. LncRNA RNCR2 is upregulated in melanomas. A) qPCR detection of RNCR2 expression in tumor tissues. B) qPCR detection of RNCR2 expression in HEMa-LP, A375, MUM2B, B16, SK-MEL-1, and A2058 cell lines. ${ }^{* *} \mathrm{p}<0.01,{ }^{* * *} \mathrm{p}<0.001$ 

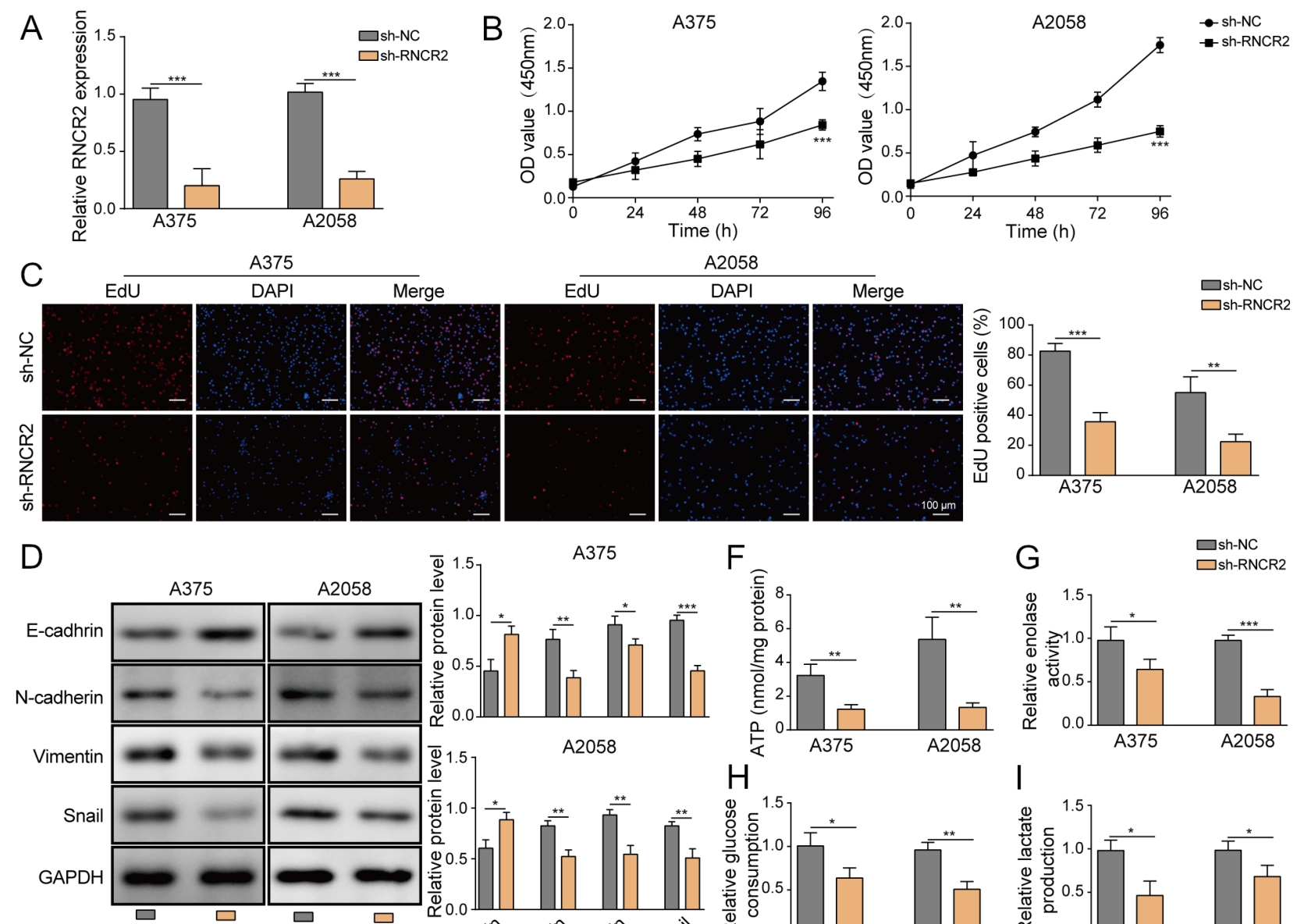

$\mathrm{E}$

$$
\text { A375 }
$$

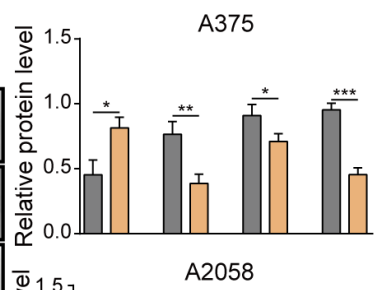

$F$
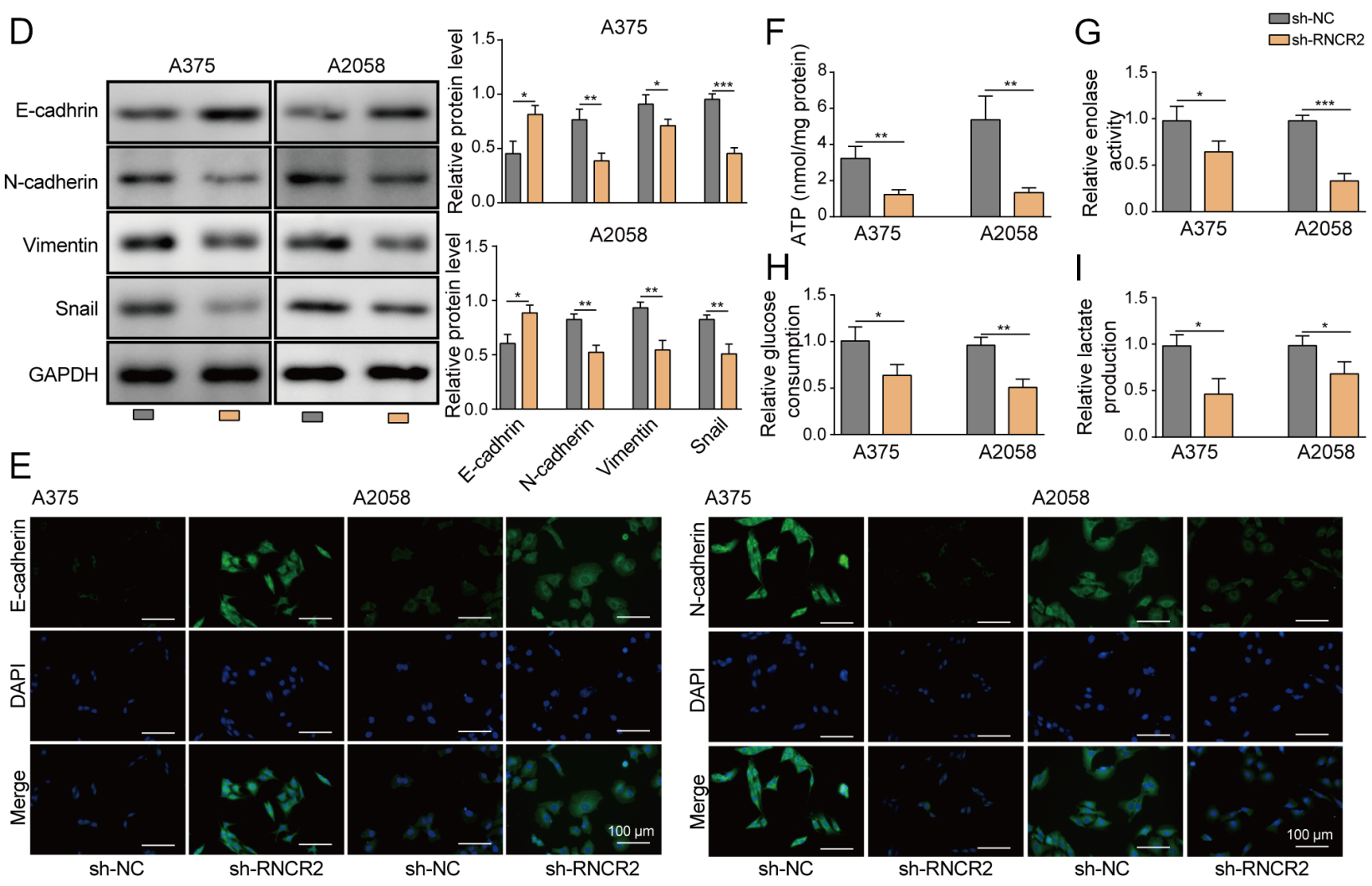

A375

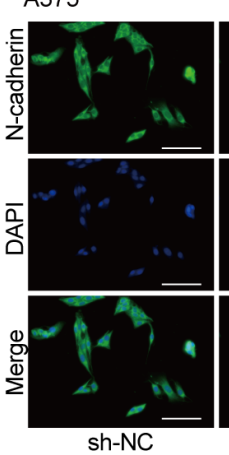

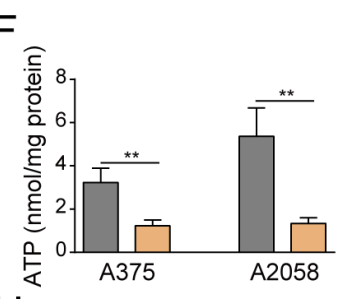

G

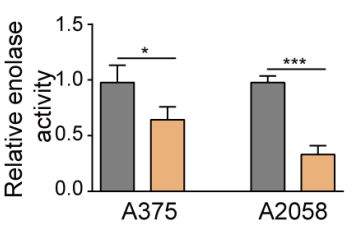

$\mathrm{H}$
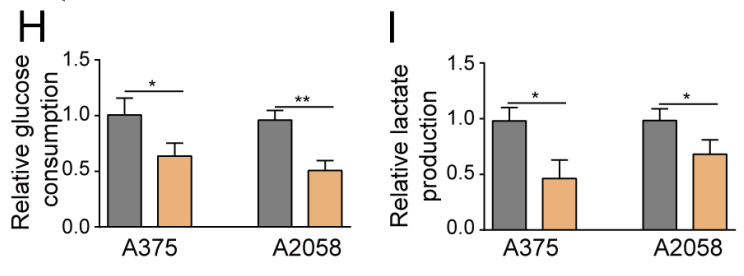

A2058
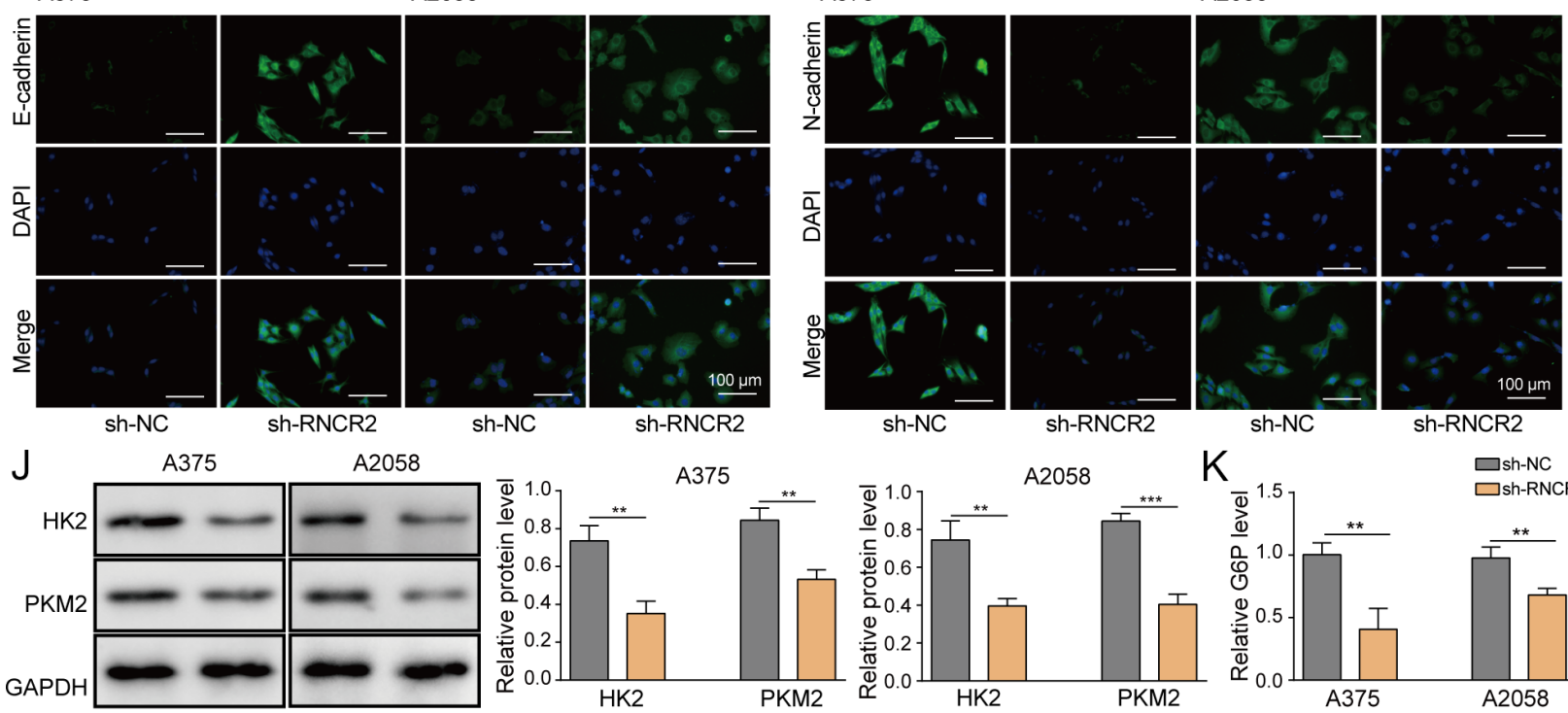

K

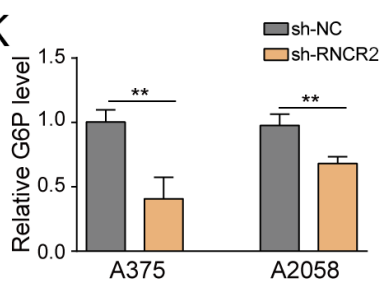

Figure 2. RNCR2 silencing inhibits melanoma cell proliferation and EMT via modulating glycolysis. A) qPCR detection of RNCR2 expression. B) CCK-8 assay was performed to assess cell viability. C) Cell proliferation was assessed via EdU assay. D) Western blot detection of N-cadherin, E-cadherin, Snail, and Vimentin expression levels. E) Immunofluorescence measurement of N-cadherin and E-cadherin expression. F-I) Detection of ATP level, enolase activity, glucose consumption, lactic acid production. J) Western blot was used for examination of the expression of HK2 and PKM2. K) The level of G6P in cells was evaluated using a G6P Assay Kit. ${ }^{*} \mathrm{p}<0.05,{ }^{* *} \mathrm{p}<0.01,{ }^{* * *} \mathrm{p}<0.001$ 
detection also proved this finding (Figure 2E). Further, we explored whether lncRNA RNCR2 regulated the glycolysis of melanoma cells. The results implied that the ATP levels, lactate production, glucose consumption, and enolase activity in A375 and A2058 cells were all suppressed by the RNCR2 knockdown in comparison with control (Figures 2F-2I). Meanwhile, HK2, PKM2, and G6P levels were also decreased in cells transfected with sh-RNCR2, indicating RNCR2 repression inhibited glycolysis (Figures 2J, 2K). Collectively, RNCR2 silencing suppresses melanoma cell glycolysis, thereby inhibiting cell proliferation and EMT.

LncRNA RNCR2 acts as a molecular sponge of miR-495-3p in melanoma cells. Firstly, an online software starBase was utilized to predict the possible target of RNCR2. It was found that RNCR2 contained the complementary sequences of miR-495-3p, suggesting RNCR2 may bind to and regulate miR-495-3p level (Figure 3A). Therefore, we used the luciferase reporter assay and RIP experiments to verify the binding relationship between miR-495-3p and RNCR2. Overexpression of miR-495-3p decreased the RNCR2-WT reporter luciferase activity, but it had a very limited effect on the luciferase activity of RNCR2-MUT reporter (Figure 3B). RIP analysis showed that Ago2 was greatly enriched in RNCR2 and miR-495-3p compared to control IgG (Figure 3C). Additionally, we found that miR-495-3p expression was downregulated in melanoma tissues and was negatively correlated with RNCR2 expression (Figures 3D-3E). Next, the downregulation of miR-495-3p was conformed in A375 and A2058 cells (Figure 3F). Furthermore, RNCR2 repression could increase miR-495-3p expression (Figure 3G). Together, RNCR2 markedly suppresses miR-495-3p expression as a molecular sponge in melanoma cells.

LncRNA RNCR2/miR-495-3p modulates HK2 expression. To elucidate the correlation between miR-495-3p and HK2 in melanoma cells, bioinformatics analysis was performed to predict potential targets. The predicted binding sites between HK2 and miR-495-3p were shown in Figure 4A. In addition, miR-495-3p overexpression distinctly reduced the HK2-WT reporter's luciferase activity, but the HK2-MUT reporter's luciferase activity was limitedly affected (Figure 4B). Subsequently, HK2 was revealed to be highly expressed in melanoma tumor tissues, which was negatively correlated with miR-495-3p level and positively correlated with RNCR2 expression (Figures 4C-4E). qPCR and western blot assays showed that $\mathrm{HK} 2$ was upregulated in melanoma cell lines, and inhibiting miR-495-3p significantly upregulated HK2 level, while the RNCR2 knockdown suppressed its expression (Figures $4 \mathrm{~F}-4 \mathrm{H}$ ). All data indicated that $\mathrm{HK} 2$ is targeted by miR-495-3p and lncRNA RNCR2 regulates HK2 expression through interacting with miR-495-3p.
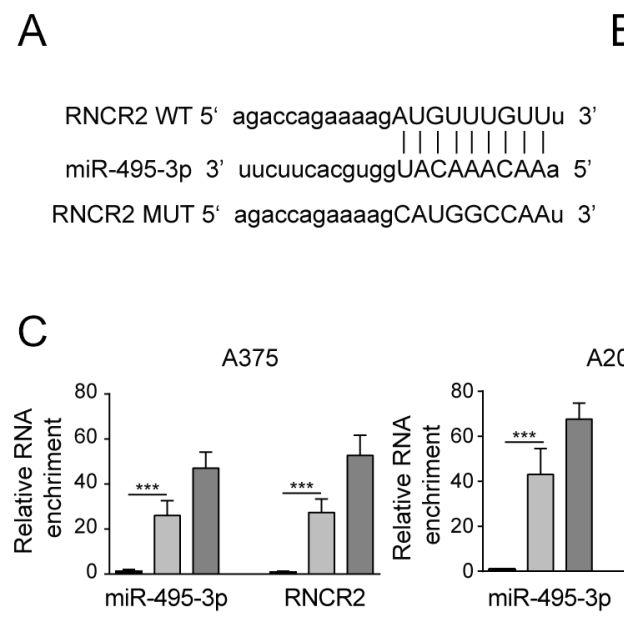

$\mathrm{F}$

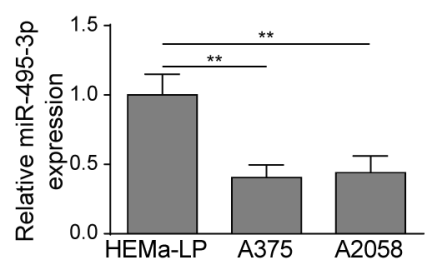

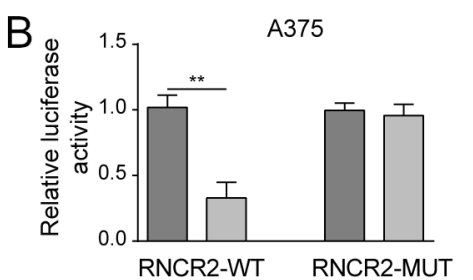

D

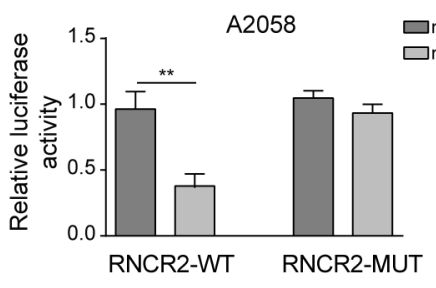

$\mathrm{E}$

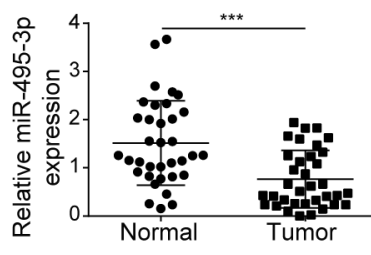

G

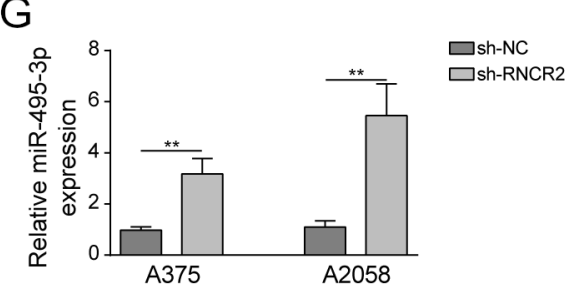

Figure 3. LncRNA RNCR2 sponges miR-495-3p in melanoma cells. A) starBase predicted the binding site of RNCR2 and miR-495-3p. B, C) Luciferase and RIP assays were performed to verify the binding relationship between RNCR2 and miR-495-3p. D) qPCR detection of miR-495-3p level. E) Correlation analysis of RNCR2 and miR-495-3p in melanoma tissues. F) qPCR detection of miR-495-3p expression in melanoma cell lines. G) qPCR measurement of miR-495-3p level in melanoma cell lines transfected with sh-NC or sh-RNCR2. ${ }^{* *} \mathbf{p}<0.01,{ }^{* * *} \mathbf{p}<0.001$ 
Inhibition of miR-495-3p or overexpression of HK2 reverses the regulation of sh-RNCR2 on glycolysis, proliferation, and EMT of melanoma cells. Next, we aimed to further confirm whether RNCR2 mediated melanoma cell proliferation, EMT, and glycolysis via miR-495-3p/ HK2 axis. Inhibition of miR-495-3p or overexpression of HK2 can reverse the suppressive effect of RNCR2 silencing on cell growth to a certain extent (Figure 5A). E-cadherin in the RNCR2 knockdown cells was remarkably decreased by miR-495-3p inhibition or HK2 overexpression, while $\mathrm{N}$-cadherin expression was notably increased (Figure 5B). ATP levels, glucose consumption, and lactate production, which were suppressed by the RNCR2 knockdown, were significantly increased under the effect of miR-495-3p inhibition or $\mathrm{HK} 2$ overexpression (Figures 5C-5E). The above results indicated that RNCR2 regulates melanoma cell proliferation and EMT via HK-2 mediated Warburg effect by targeting miR-495-3p.

Knockdown of lncRNA RNCR2 inhibits melanoma tumor growth. In order to investigate whether RNCR2 would affect the growth of melanoma, a subcutaneous tumorigenesis model was established, and tumor volume and weight were analyzed. The tumor size formed by A375/sh-RNCR2 cells was significantly smaller than that of A375/sh-NC, demonstrating RNCR2 shRNA significantly alleviated the growth of the tumor (Figures 6A, 6B). Consistently, the tumor formed by A375/sh-RNCR2 cells was remarkably lighter than that of A375/sh-NC, suggesting that RNCR2 suppression markedly
A HK2 WT 5' agGgGgaguUUUUAgUUUgUUu 3' miR-495-3p 3' uUCUUCACGUGG UACAAACAAa 5' HK2 WT 5' agCUUGAUGUGGUAGUUGACCa 3
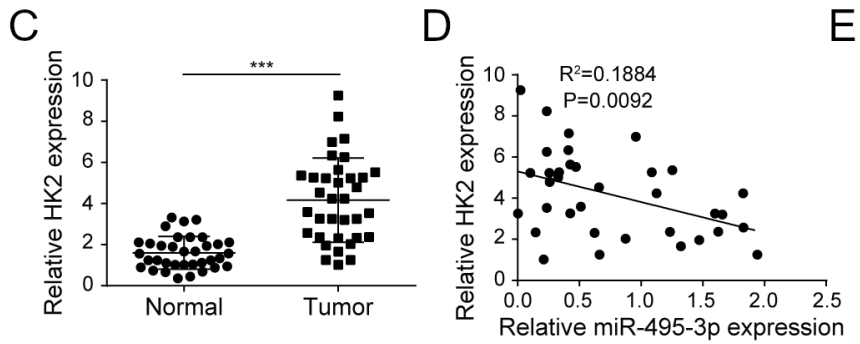

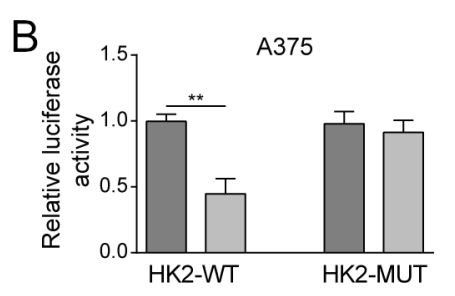

$\mathrm{E}$

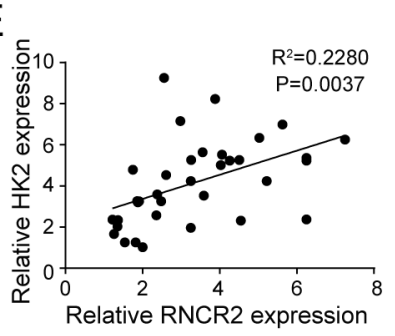

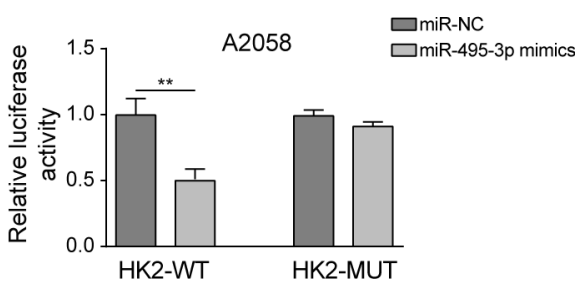

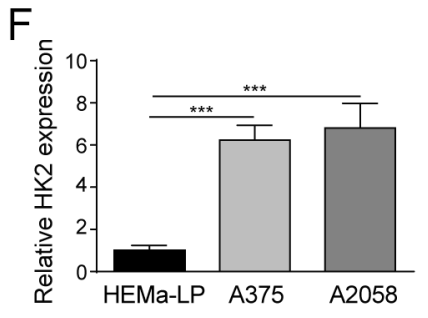

\section{G}

$$
\begin{aligned}
& \square \text { inhibitor NC } \square \text { miR-495-3p inhibitor+sh-NC } \\
& \square \text { miR-495-3p inhibitor } \\
& \text { miR-495-3p inhibitor+sh-RNCR }
\end{aligned}
$$

miR-495-3p inhibitor+sh-RNCR2
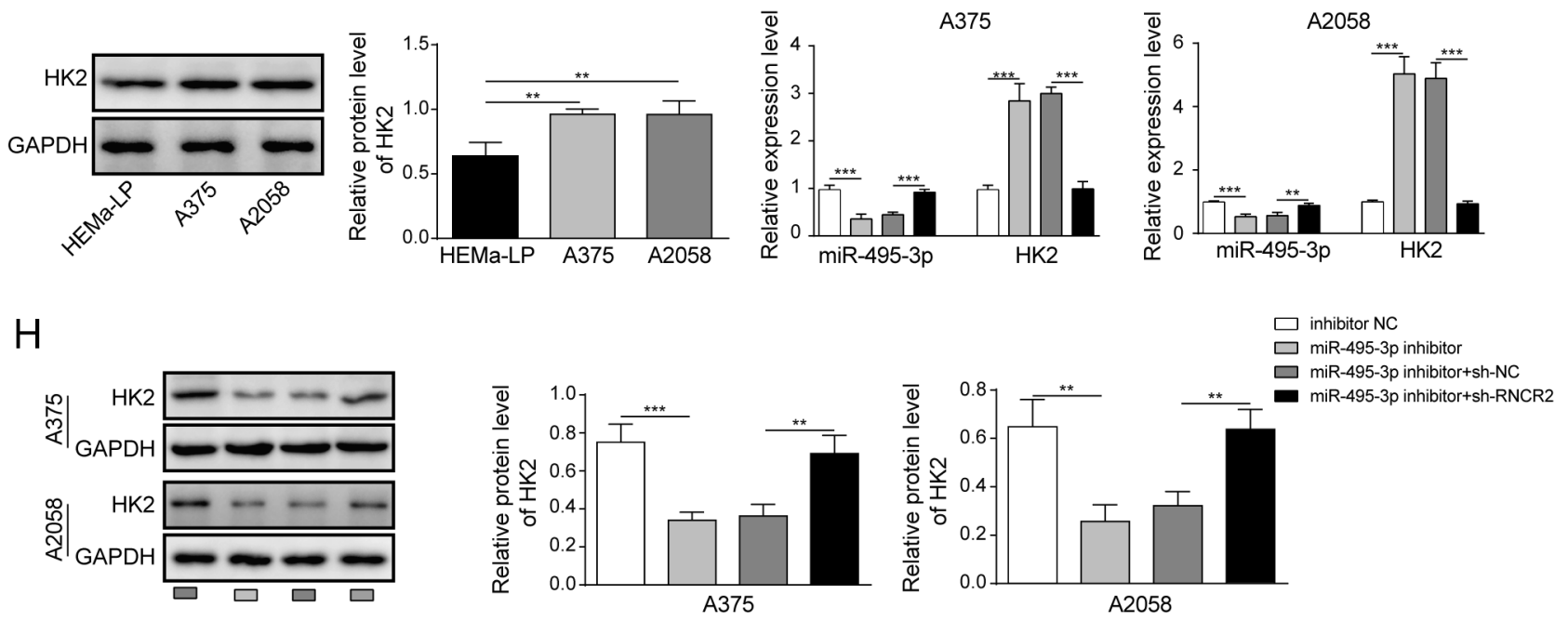

Figure 4. LncRNA RNCR2/miR-495-3p modulates HK2 expression. A) starBase predicted the binding site of HK2 and miR-495-3p. B) Luciferase assay was used to verify the binding relationship between HK2 and miR-495-3p. C) qPCR detection of miR-495-3p expression in tumor tissues. D) Correlation detection between $\mathrm{HK} 2$ and miR-495-3p in melanoma tissues. E) Correlation analysis of RNCR2 and HK2 expression in melanoma tissues. F) qPCR/western blot measurement of HK2 level in melanoma cell lines. G, H) qPCR/western blot detection of the effect of RNCR2/miR-495-3p on HK2 expression. ${ }^{* *} \mathrm{p}<0.01,{ }^{* *} \mathrm{p}<0.001$ 

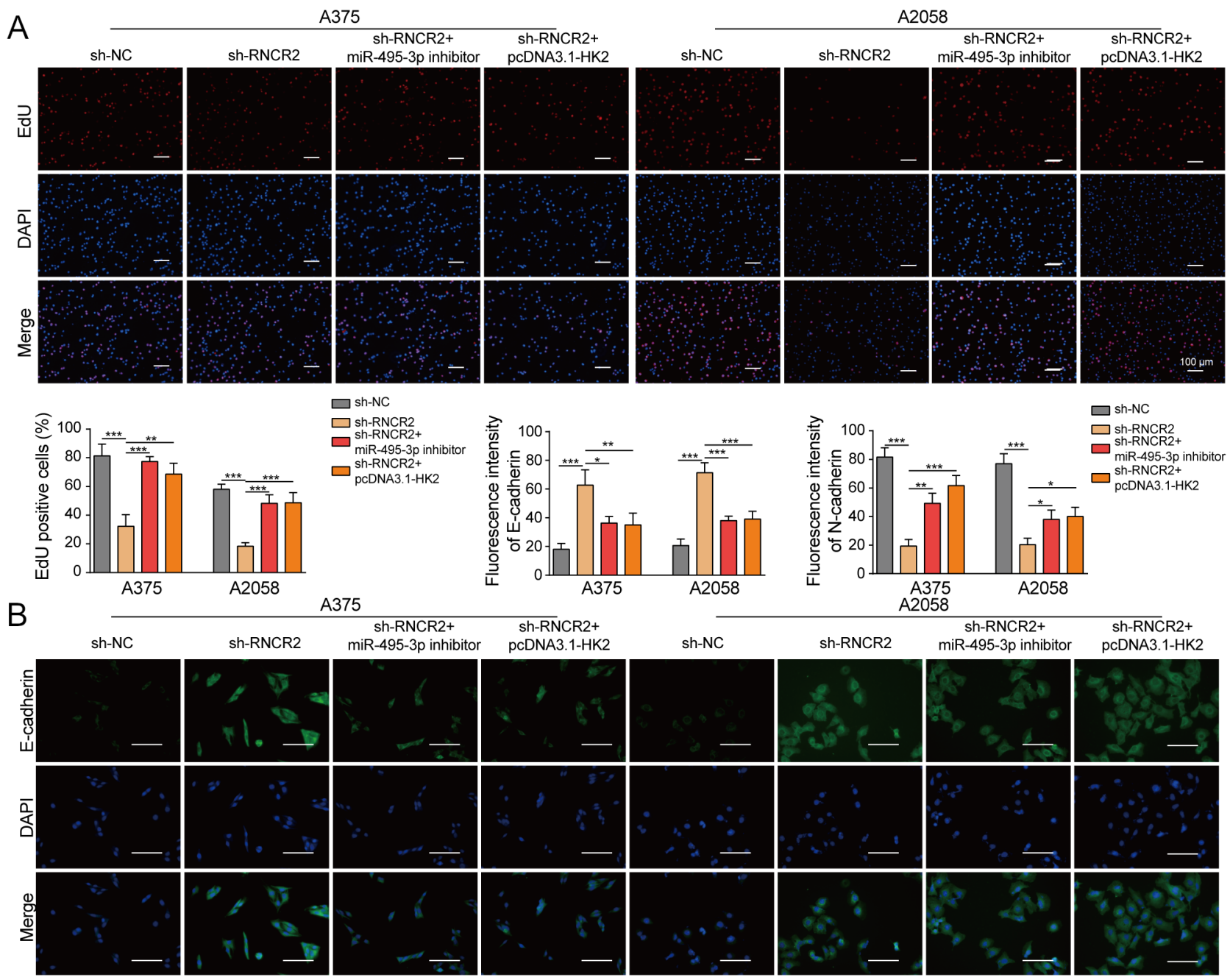
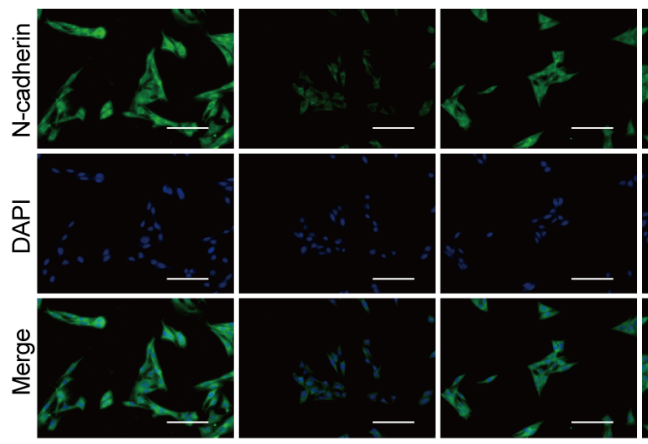

C

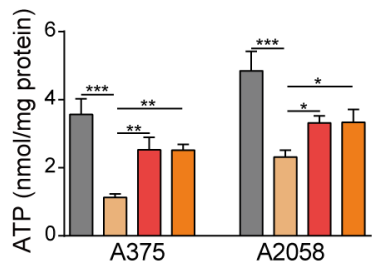

$\mathrm{D}$

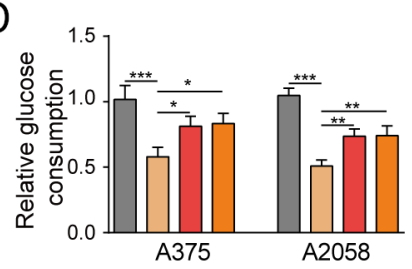

E

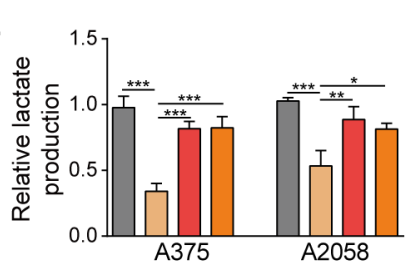

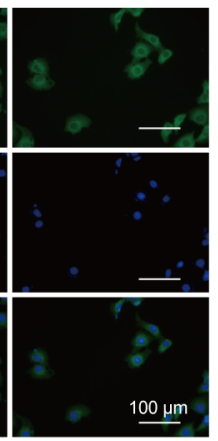

$\square$ sh-NC

$\square$ sh-RNCR2

$\square$ miR-495-3p inhibito

$\square$ sh-RNCR2+

Figure 5. Inhibition of miR-495-3p or overexpression of HK2 reverses the regulation of sh-RNCR2 on glycolysis, proliferation, and EMT of melanoma cells. A) EdU assay was used to detect cell proliferation. B) Immunofluorescence detection of E-cadherin and N-cadherin expression. C) Enhanced ATP Assay Kit was used to detect ATP level. D) Glucose Assay Kit was used to measure glucose consumption. E) Lactate production was assessed by using a Lactate Assay Kit. ${ }^{*} \mathrm{p}<0.05,{ }^{* *} \mathrm{p}<0.01,{ }^{* *} \mathrm{p}<0.001$ 
reduced tumor weight (Figure $6 \mathrm{C}$ ). Then, miR-495-3p and HK2 expression were measured in tumor tissues (Figures 6D, 6E). Certainly, miR-495-3p was upregulated, while HK2 was inactivated by RNCR2 deficiency. These results indicate that RNCR2 targets miR-495-3p to modulate HK2 expression and hampered tumor growth in melanoma.

\section{Discussion}

Studies showed that the prognosis of advanced melanoma has not been significantly improved due to the resistance to chemotherapy and the lack of targeted therapy [32]. In recent years, studies on the treatment of melanoma have focused on exploring novel therapeutic targets and strategies [33]. It was reported that IncRNA RNCR2 is abnormally expressed in tumors and involved in cancer progression [34]. In this study, lncRNA RNCR2 is upregulated in melanoma tumors, which is consistent with published findings. Functional experiments confirmed that RNCR2 knockdown inhibited the cell proliferation and EMT in melanoma by repressing glycolysis via the miR-495-3p/HK2 axis.

Although the role played by lncRNAs in skin diseases is not yet clear, more and more abnormally expressed lncRNAs have been discovered in hyperproliferative skin diseases recently [35]. Melanoma is regarded by highly invasive and metastatic characteristics $[36,37]$. Because of its functions in chromatin modification, transcription regulation, and RNA regulation, IncRNAs have gradually become the research focus of targeted therapy in melanoma $[35,38]$. For example, exogenous IncRNA SAMMSON enhances the proliferation ability of melanoma cells, while the knockdown of SAMMSON inhibits melanoma cell activity by regulating MAPK [39]. It has been reported that RNCR2 has a regula- tory effect on the cell cycle, which indicates that RNCR2 plays an important role in regulating the proliferation of tumor cells [40]. However, the relationship between RNCR2 and malignant melanoma has not fully been studied. This study proved that RNCR2 is highly expressed in melanoma tissues and melanoma cells, its suppression drastically inhibited cell proliferation and tumor growth. Previous studies showed that RNCR2 can significantly affect the EMT process of various tumor cells [28, 41, 42]. Similarly, knockdown of RNCR2 in this study repressed EMT evidenced by the mediation of N-cadherin, Snail, E-cadherin, and Vimentin. In addition, we found that RNCR2 silencing conspicuously reduced ATP levels, enolase activity, glucose consumption, and lactate production. HK2 is important for the aerobic pathway and is a key enzyme for G6P. The knockdown of RNCR2 also suppressed HK2, PKM2 expression, and G6P level. These data proved that RNCR2 may promote cancer development by increasing the glycolysis in melanoma.

Some ncRNAs, especially lncRNAs, bind miRNAs through complementary competition to reduce miRNA levels and miRNA activity [43]. This competitive endogenous RNA (ceRNA) hypothesis has been proven in various cancers [44, 45]. For example, RNCR2, as a miRNA sponge, combined with miR-22-3p in the form of ceRNA upregulates SIRT1 and inhibits senescence of liver cancer cells [46]. In our work, bioinformatics prediction revealed that RNCR2 could bind to miR-495-3p. miR-495 had a low expression trend in malignancies [47-49]. Consistently, miR-495-3p was inhibited by RNCR2 in melanoma tissues and melanoma cells, showing a trend of low expression. Besides, HK2 expression was positively related to RNCR2, but the expression of HK2 was directly regulated by $\mathrm{miR}-495-3 \mathrm{p}$. Hexokinase is the catalyst for the first step in glycolysis. The expression of HK2

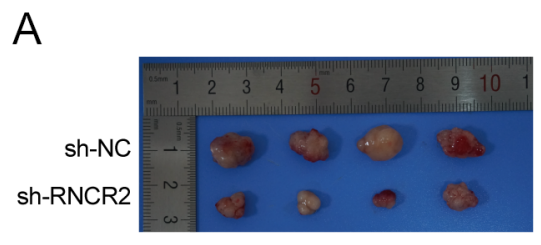

$\mathrm{D}$

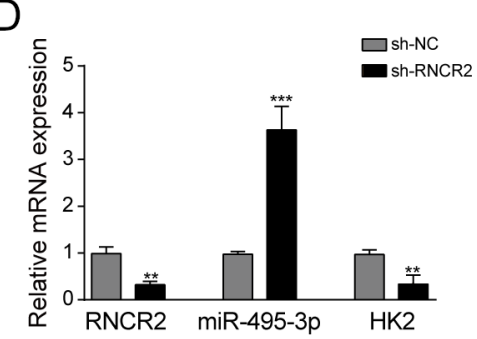

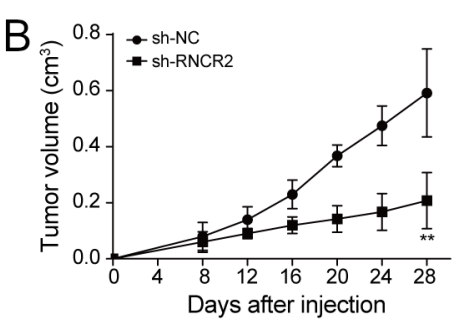

E

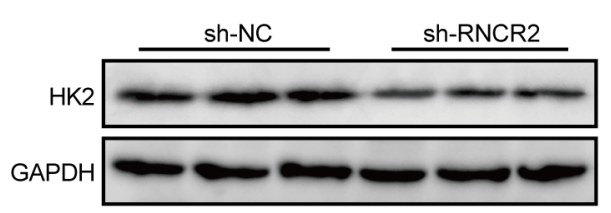

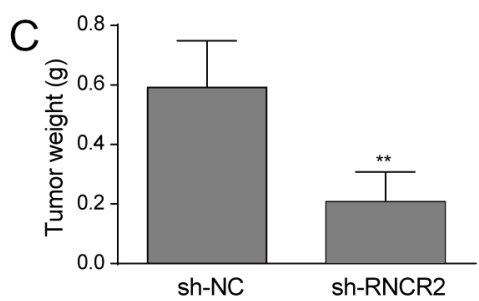

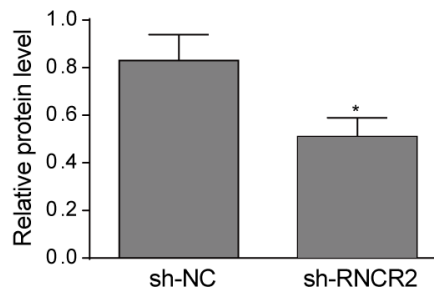

Figure 6. Knockdown of lncRNA RNCR2 inhibits melanoma tumor growth. A) Xenograft tumor tissues are presented. B) Tumor volume was monitored every 4 days. C) Tumor weight was determined after 28 days of injection. D) qPCR detection of RNCR2, miR-495-3p, HK2 expression. E) Western blot detection of HK2 expression in tumor tissues. ${ }^{*} \mathrm{p}<0.05,{ }^{* *} \mathrm{p}<0.01$ 
and smaller hexokinase 1 (HK1) were upregulated in cancer cells to drive glycolysis [50]. The high expression of HK1 and HK2 in melanoma cells also promoted the glycolysis process without doubt [51]. This study firstly demonstrated that RNCR2 targets miR-495-3p to regulate the HK2-mediated glycolysis process in melanoma.

In summary, RNCR2 was strongly expressed in melanoma tissues and cells and may act as a ceRNA for miR-495-3p, thereby promoting HK2-mediated glycolysis. Importantly, we found that after repressing RNCR2, miR-495-3p can be significantly upregulated while inhibiting HK2 to reduce glycolysis and EMT, thereby blocking tumor growth. It is foreseeable that our findings prove that RNCR2 can be used as a potential molecular target in the targeted therapy of malignant melanoma, providing a theoretical basis for exploring the effective and high-quality treatment of melanoma.

Acknowledgments: We would like to give our sincere gratitude to the reviewers for their constructive comments.

\section{References}

[1] HAASS NK, SCHUMACHER U. Melanoma never says die. Exp Dermatol 2014; 23: 471-472. https://doi.org/10.1111/ exd. 12400

[2] TROTTER SC, SROA N, WINKELMANN RR, OLENCKI T, BECHTEL M. A Global Review of Melanoma Follow-up Guidelines. J Clin Aesthet Dermatol 2013; 6: 18-26.

[3] SIEGEL RL, MILLER KD, JEMAL A. Cancer Statistics, 2017. CA Cancer J Clin 2017; 67: 7-30. https://doi.org/10.3322/ caac. 21387

[4] THYAGARAJAN A, SHABAN A, SAHU RP. MicroRNADirected Cancer Therapies: Implications in Melanoma Intervention. J Pharmacol Exp Ther 2018; 364: 1-12. https://doi. org/10.1124/jpet.117.242636

[5] MADHUNAPANTULA SV, ROBERTSON GP. Chemoprevention of melanoma. Adv Pharmacol 2012; 65: 361-398. https://doi.org/10.1016/B978-0-12-397927-8.00012-9

[6] EGGERMONT AM, ROBERT C. Melanoma: smart therapeutic strategies in immuno-oncology. Nat Rev Clin Oncol 2014; 11: 181-182. https://doi.org/10.1038/nrclinonc.2014.36

[7] CARUSO JP, COHEN-INBAR O, BILSKY MH, GERSZTEN PC, SHEEHAN JP. Stereotactic radiosurgery and immunotherapy for metastatic spinal melanoma. Neurosurg Focus 2015; 38: E6. https://doi.org/10.3171/2014.11.FOCUS14716

[8] HSU PP, SABATINI DM. Cancer cell metabolism: Warburg and beyond. Cell 2008; 134: 703-707. https://doi. org/10.1016/j.cell.2008.08.021

[9] WARBURG O. On the origin of cancer cells. Science 1956; 123: 309-314. https://doi.org/10.1126/science.123.3191.309

[10] LIBERTI MV, LOCASALE JW. The Warburg Effect: How Does it Benefit Cancer Cells? Trends Biochem Sci 2016; 41: 211-218. https://doi.org/10.1016/j.tibs.2015.12.001
[11] LU J, TAN M, CAI Q. The Warburg effect in tumor progression: mitochondrial oxidative metabolism as an anti-metastasis mechanism. Cancer Lett 2015; 356: 156-164. https:// doi.org/10.1016/j.canlet.2014.04.001

[12] DEBERARDINIS RJ, LUM JJ, HATZIVASSILIOU G, THOMPSON CB. The biology of cancer: metabolic reprogramming fuels cell growth and proliferation. Cell Metab 2008; 7: 11-20.

[13] ZHAO H, DUAN Q, ZHANG Z, LI H, WU H et al. Up-regulation of glycolysis promotes the stemness and EMT phenotypes in gemcitabine-resistant pancreatic cancer cells. J Cell Mol Med 2017; 21: 2055-2067. https://doi.org/10.1111/ jcmm.13126

[14] OU J, MIAO H, MA Y, GUO F, DENG J et al. Loss of abhd5 promotes colorectal tumor development and progression by inducing aerobic glycolysis and epithelial-mesenchymal transition. Cell Rep 2014; 9: 1798-1811. https://doi. org/10.1016/j.celrep.2014.11.016

[15] LI X, CAO Y, GONG X, LI H. Long noncoding RNAs in head and neck cancer. Oncotarget 2017; 8: 10726-10740. https://doi.org/10.18632/oncotarget.12960

[16] MCANINCH D, ROBERTS CT, BIANCO-MIOTTO T. Mechanistic Insight into Long Noncoding RNAs and the Placenta. Int J Mol Sci 2017; 18:1371. https://doi.org/10.3390/ ijms 18071371

[17] BENETATOS L, VOULGARIS E, VARTHOLOMATOS G. The crosstalk between long non-coding RNAs and PI3K in cancer. Med Oncol 2017; 34: 39. https://doi.org/10.1007/ s12032-017-0897-2

[18] SINGH DK, PRASANTH KV. Functional insights into the role of nuclear-retained long noncoding RNAs in gene expression control in mammalian cells. Chromosome Res 2013; 21: 695-711. https://doi.org/10.1007/s10577-013-9391-7

[19] LU Q, ZHAO N, ZHA G, WANG H, TONG Q et al. LncRNA HOXA11-AS Exerts Oncogenic Functions by Repressing p21 and miR-124 in Uveal Melanoma DNA. Cell Biol 2017; 36: 837-844. https://doi.org/10.1089/dna.2017.3808

[20] LI J, CHEN Y, CHEN Z, HE A, XIE H et al. SPRY4-IT1: A novel oncogenic long non-coding RNA in human cancers. Tumour Biol 2017; 39: 1010428317711406. https://doi. org/10.1177/1010428317711406

[21] ZHAO H, XING G, WANG Y, LUO Z, LIU G et al. Long noncoding RNA HEIH promotes melanoma cell proliferation, migration and invasion via inhibition of miR-200b/a/429. Biosci Rep 2017; 37: BSR20170682. https://doi.org/10.1042/ BSR20170682

[22] LV L, JIA JQ, CHEN J. The IncRNA CCAT1 Upregulates Proliferation and Invasion in Melanoma Cells via Suppressing miR-33a. Oncol Res 2018; 26: 201-208. https://doi.org/1 0.3727/096504017X14920318811749

[23] WANG BJ, DING HW, MA GA. Long Noncoding RNA PVT1 Promotes Melanoma Progression via Endogenous Sponging miR-26b. Oncol Res 2018; 26: 675-681. https://doi. org/10.3727/096504017X14920318811730

[24] LI X, ZHANG L, SONG P, XU J, LI G. Long non-coding RNA PANDAR promotes melanoma cell invasion through regulating epithelial-mesenchymal transition. Int J Clin Exp Pathol 2018; 11: 2430-2439. 
[25] ISHII N, OZAKI K, SATO H, MIZUNO H, SUSUMU S et al. Identification of a novel non-coding RNA, MIAT, that confers risk of myocardial infarction. J Hum Genet 2006; 51: 1087-1099. https://doi.org/10.1007/s10038-006-0070-9

[26] MERCER TR, QURESHI IA, GOKHAN S, DINGER ME, LI $\mathrm{G}$ et al. Long noncoding RNAs in neuronal-glial fate specification and oligodendrocyte lineage maturation. BMC Neurosci 2010; 11: 14. https://doi.org/10.1186/1471-2202-11-14

[27] RAPICAVOLI NA, POTH EM, BLACKSHAW S. The long noncoding RNA RNCR2 directs mouse retinal cell specification. BMC Dev Biol 2010; 10: 49. https://doi. org/10.1186/1471-213X-10-49

[28] ZHU L, WANG Y, YANG C, LI Y, ZHENG Z et al. Long non-coding RNA MIAT promotes the growth of melanoma via targeting miR-150. Hum Cell 2020; 33: 819-829. https:// doi.org/10.1007/s13577-020-00340-y

[29] YANG Y, ZHANG Z, WU Z, LIN W, YU M. Downregulation of the expression of the lncRNA MIAT inhibits melanoma migration and invasion through the PI3K/AKT signaling pathway. Cancer Biomark 2019; 24: 203-211. https://doi. org/10.3233/CBM-181869

[30] GAO F, LI M, LIU WB, ZHOU ZS, ZHANG R et al. Epigallocatechin gallate inhibits human tongue carcinoma cells via HK2mediated glycolysis. Oncol Rep 2015; 33: 1533-1539. https://doi.org/10.3892/or.2015.3727

[31] LU J, LIANG X, GAO Y, FU G, SHEN Q. Hexokinase2 controls angiogenesis in melanoma by promoting aerobic glycolysis and activating the p38-MAPK signaling. J Cell Biochem 2019; 120: 19721-19729. https://doi.org/10.1002/jcb.29278

[32] JOHNSON DB, SOSMAN JA. Therapeutic Advances and Treatment Options in Metastatic Melanoma. JAMA Oncol 215; 1: 380-386. https://doi.org/10.1001/jamaoncol.2015.0565

[33] DAVEY RJ, VAN DER WESTHUIZEN A, BOWDEN NA. Metastatic melanoma treatment: Combining old and new therapies. Crit Rev Oncol Hematol 2016; 98: 242-253. https://doi.org/10.1016/j.critrevonc.2015.11.011

[34] DA CM, GONG CY, NAN W, ZHOU KS, WU ZL et al. The role of long non-coding RNA MIAT in cancers. Biomed Pharmacother 2020; 129: 110359. https://doi.org/10.1016/j. biopha.2020.110359

[35] TANG L, LIANG Y, XIE H, YANG X, ZHENG G. Long non-coding RNAs in cutaneous biology and proliferative skin diseases: Advances and perspectives. Cell Prolif 2020; 53: e12698. https://doi.org/10.1111/cpr.12698

[36] LI Z, YU X, SHEN J, JIANG Y. MicroRNA dysregulation in uveal melanoma: a new player enters the game. Oncotarget 2015; 6: 4562-4568. https://doi.org/10.18632/oncotarget. 2923

[37] MILLER AJ, MIHM MC, JR. Melanoma. N Engl J Med 2006; 355: 51-65. https://doi.org/10.1056/NEJMra052166

[38] YU X, ZHENG H, TSE G, CHAN MT, WU WK. Long noncoding RNAs in melanoma. Cell Prolif 2018; 51: e12457. https://doi.org/10.1056/NEJMra052166

[39] LEUCCI E, VENDRAMIN R, SPINAZZI M, LAURETTE P, FIERS $M$ et al. Melanoma addiction to the long non-coding RNA SAMMSON. Nature 2016; 531: 518-522. https://doi. org/10.1038/nature17161
[40] YAN B, YAO J, LIU JY, LI XM, WANG XQ et al. lncRNAMIAT regulates microvascular dysfunction by functioning as a competing endogenous RNA. Circ Res 2015; 116: 11431156. https://doi.org/10.1161/CIRCRESAHA.116.305510

[41] MITRA R, CHEN X, GREENAWALT EJ, MAULIK U, JIANG $\mathrm{W}$ et al. Decoding critical long non-coding RNA in ovarian cancer epithelial-to-mesenchymal transition. Nat Commun 2017; 8: 1604. https://doi.org/10.1038/s41467017-01781-0

[42] ZHONG W, XU Z, WEN S, XIE T, WANG F et al. Long noncoding RNA myocardial infarction associated transcript promotes epithelial-mesenchymal transition and is an independent risk factor for poor prognosis of tongue squamous cell carcinoma. J Oral Pathol Med 2019; 48: 720-727. https://doi. org/10.1111/jop.12892

[43] SALMENA L, POLISENO L, TAY Y, KATS L, PANDOLFI PP. A ceRNA hypothesis: the Rosetta Stone of a hidden RNA language? Cell 2011; 146: 353-358. https://doi.org/10.1016/j. cell.2011.07.014

[44] WANG L, CHO KB, LI Y, TAO G, XIE Z et al. Long Noncoding RNA (lncRNA)-Mediated Competing Endogenous RNA Networks Provide Novel Potential Biomarkers and Therapeutic Targets for Colorectal Cancer. Int J Mol Sci 2019; 20: 5758. https://doi.org/10.3390/ijms20225758

[45] FANG X, ZHANG J, LI C, LIU J, SHI Z et al. Long non-coding RNA SNHG22 facilitates the malignant phenotypes in triple-negative breast cancer via sponging miR-324-3p and upregulating SUDS3. Cancer Cell Int 2020; 20: 252. https:// doi.org/10.1186/s12935-020-01321-9

[46] ZHAO L, HU K, CAO J, WANG P, LI J et al. lncRNA miat functions as a ceRNA to upregulate sirtl by sponging miR22-3p in HCC cellular senescence. Aging (Albany NY) 2019; 11: 7098-7122. https://doi.org/10.18632/aging.102240

[47] CAO Y, XIONG JB, ZHANG GY, LIU Y, JIE ZG et al. Long Noncoding RNA UCA1 Regulates PRL-3 Expression by Sponging MicroRNA-495 to Promote the Progression of Gastric Cancer. Mol Ther Nucleic Acids 2020; 19: 853-864. https://doi.org/10.1016/j.omtn.2019.10.020

[48] ALQURASHI N, HASHIMI SM, ALOWAIDI F, IVANOVSKI S, FARAG A et al. miR-496, miR-1185, miR-654, miR-3183 and miR-495 are downregulated in colorectal cancer cells and have putative roles in the mTOR pathway. Oncol Lett 2019; 18: 1657-1668. https://doi.org/10.3892/ ol.2019.10508

[49] YIN G, LIU Z, WANG Y, SUN L, WANG L et al. ZNF503 accelerates aggressiveness of hepatocellular carcinoma cells by down-regulation of GATA3 expression and regulated by microRNA-495. Am J Transl Res 2019; 11: 3426-3437.

[50] KOCH A, EBERT EV, SEITZ T, DIETRICH P, BERNEBURG $M$ et al. Characterization of glycolysis-related gene expression in malignant melanoma. Pathol Res Pract 2020; 216: 152752. https://doi.org/10.1016/j.prp.2019.152752

[51] KUDRYAVTSEVA AV, FEDOROVA MS, ZHAVORONKOV A, MOSKALEV AA, ZASEDATELEV AS et al. Effect of lentivirus-mediated shRNA inactivation of HK1, HK2, and HK3 genes in colorectal cancer and melanoma cells. BMC Genet 2016; 17: 156. https://doi.org/10.1186/ s12863-016-0459-1 